\title{
Avaliação do perfil hematológico, bioquímico e esfregaço de sangue periférico com vistas ao perfil sanitário em primatas do gênero Cebus mantidos em cativeiro
}

\author{
Evaluation of hematological profile, biochemical and peripheral \\ blood smear with a view to the health profile in primates of the \\ Cebus genre maintained in captivity
}

\author{
David Baruc Cruvinel Lima ${ }^{1 *}$; Karynne Marques Mesquita dos Santos ${ }^{2}$; \\ Hatawa Melo de Almeida ${ }^{3}$; Caliandra Bona Nascimento ${ }^{3}$; \\ Aírton Mendes Conde Júnior ${ }^{4}$; Márcia dos Santos Rizzo ${ }^{4}$
}

\begin{abstract}
Resumo
Os primatas não humanos atuam como modelo para estudos sobre a dinâmica das vias de transmissão e história natural de doenças compartilhadas entre homens e animais. $\mathrm{O}$ ambiente de cativeiro é propício à disseminação de doenças de caráter zoonótico. Muitos destes animais não apresentam sintomatologia clínica, mesmo estando infectados, o que os caracterizam como importante fonte de infecção para os animais domésticos e o homem. O objetivo deste trabalho foi avaliar a sanidade de primatas não humanos mantidos em cativeiro através de análise hematológica e bioquímica, bem como esfregaço de sangue periférico, com intuito de investigar a presença de patógenos de caráter zoonótico, servindo de modelo para estudos futuros sobre a dinâmica das vias de transmissão de doenças compartilhadas entre homens e animais. Foram coletadas amostras de sangue de 15 macacos prego (Cebus sp.), adultos, clinicamente saudáveis e pertencentes ao Parque Zoobotânico de Teresina. Foram coradas lâminas de esfregaço sanguíneo e obtidos os perfis hematológicos e bioquímicos de cada animal. A análise dos dados baseou-se em estatística básica. Não foi observado nenhum hemoparasita presente em sangue periférico. Todos os animais estavam anêmicos, $46,7 \%$ trombopênicos e $87 \%$ apresentavam algum tipo de processo patológico de evolução crônica, devido à elevada taxa de monócitos encontrados. Todos os animais apresentaram elevadas taxas de fosfatase alcalina e das transaminases AST e ALT, indicando injúria do parênquima hepático. Novos estudos deverão ser realizados para melhor elucidação dos resultados, visto que dados bioquímicos fisiológicos de primatas do gênero Cebus são escassos na literatura.
\end{abstract}

Palavras-chave: Primatas, zoonoses, hematologia, perfil bioquímico

\begin{abstract}
Non-human primates serve as a model for studies on the dynamics of transmission routes and natural history of diseases shared between humans and animals. The captive environment is conducive the dissemination of zoonotic diseases. Many of these animals do not present clinical symptomatology,
\end{abstract}

\footnotetext{
${ }^{1}$ Discente de Graduação em Medicina Veterinária, Universidade Federal do Piauí, UFPI, Teresina, PI. E-mail: davidbaruc@, ig.com.br

2 Médica Veterinária, UFPI, Teresina, PI. E-mail: karynne_medvet@hotmail.com

${ }^{3}$ Discentes de Doutorado em Ciência Animal, UFPI, Teresina, PI. E-mail: hatawama@yahoo.com.br; criarcentroveterinario@ hotmail.com

${ }^{4}$ Profs. Drs., UFPI, Teresina, PI. E-mail: airtoncondejr@uol.com.br; marciarizzo@ufpi.edu.br

* Autor para correspondência
} 
even when infected, which characterize them as an important source of infection for domestic animals and man. The objective of this study was to evaluate the sanity of non-human primates kept in captivity through hematological and biochemical analysis as well as peripheral blood smear, aiming to investigate the presence of zoonotic pathogens, serving as a model for future studies on the dynamics of routes of transmission of diseases shared between humans and animals. Were collected samples of blood from 15 nail monkeys (Cebus sp.), adults, clinically healthy and belonging to Park Zoobotanic of Teresina. Were stained smear sanguine blades and obtained the haematological and biochemical profiles of each animal. The data analysis was based on basic statistics. Did not observed any haemoparasite present in peripheral blood. All animals were anemic, $46,7 \%$ thrombopenics and $87 \%$ of the animals showed some type of pathological process of chronic evolution, due to the high rate of monocytes found. All animals showed high rates of alkaline phosphatase, and transaminases AST and ALT, indicating injury of the hepatic parenchyma. New studies should be conducted to better elucidate of results, seeing that biochemical physiological data primate of the genus Cebus are scarce in literature.

Key words: Primates, zoonosis, hematology, biochemical profile

\section{Introdução}

Oestudo das populações de primatas não humanos tem gerado informações a respeito das zoonoses negligenciadas e emergentes. Sendo membros de um habitat biologicamente diversificado, estes animais atuam como sentinelas para investigação de inúmeros patógenos, servindo de modelo para estudos sobre a dinâmica das vias de transmissão e história natural de doenças compartilhadas entre homens e animais (WOLFE et al., 1998).

$\mathrm{O}$ avanço da agricultura e da pecuária próximo às áreas naturais proporcionou o contato da população humana e de seus animais domésticos com as populações de animais silvestres e seus respectivos habitats, facilitando a disseminação de agentes infecciosos e parasitários para novos hospedeiros e ambientes (CORRÊA; PASSOS, 2001). Estas ações não só modificaram o ambiente, mas também alteraram a virulência, patogenicidade, distribuição e o rol de hospedeiros afetados por agentes infecciosos, que passaram a ter o potencial de causar epidemias (ou mesmo pandemias) graves com grande mortalidade, tanto na espécie humana como em espécies de animais domésticos, de produção ou silvestres. Com isso, estabeleceramse novas relações entre hospedeiros e parasitas, e novos nichos ecológicos na cadeia de transmissão das doenças (MONTEIRO, 2008; NASPHV, 2008).

Neste sentido, o ambiente de cativeiro é propício à disseminação de doenças de caráter zoonótico, apesar do rigoroso manejo sanitário observado em zoológicos. A proximidade de visitantes e tratadores com animais silvestres favorece o contato do homem, e vice-versa, com inúmeros patógenos infecciosos, sejam eles exóticos ou não para a espécie em questão (LEITE et al., 2008; CDC, 2009). Ademais, os animais de cativeiro, por estarem infectados, podem se comportar como transmissores de parasitas para o meio ambiente. Vários estudos têm demonstrado que primatas não humanos são naturalmente infectados por parasitos que são patogênicos para o homem (CARMO; SALGADO, 2003; AMÂNCIO et al., 2008). Vale salientar que grande parte destes animais não apresenta sintomatologia clínica, mesmo estando infectados com agentes infecciosos, o que os tornam importante fonte de infecção para os animais domésticos e o homem (ACHA; SZYFRES, 2003).

Portanto, devido ao caráter zoonótico de inúmeras doenças comuns entre o homem e primatas, e pelo fato destes animais poderem ser reservatórios de patógenos que infectam seres humanos nos faz concluir que estudos com primatas têm uma relevância inquestionável. Além disso, o estudo destas zoonoses poderá auxiliar na implantação de ações que possam monitorar a sanidade destes animais, bem como nas medidas de prevenção e controle para doenças em animais e no homem (BURGOS-RODRIGUES, 2011). 
Dados hematológicos e bioquímicos de primatas não humanos são escassos na literatura, o que tem impulsionado estudos a fim de estabelecer limites para parâmetros saúde-doença, assim como a estruturação de dados para compreensão das enfermidades presentes nestes animais e as mudanças ocasionadas por patógenos (RIVIELLO; WIRZ, 2001).

Diante do exposto, o objetivo deste trabalho foi avaliar a sanidade de primatas não humanos mantidos em cativeiro por meio de análise hematológica e bioquímica, além da análise de esfregaço de sangue periférico, com o intuito de investigar a presença de patógenos de caráter zoonótico.

\section{Material e Métodos}

Foram utilizados para este estudo animais do gênero Cebus sp, adultos (seis machos e nove fêmeas), clinicamente saudáveis e pertencentes ao Parque Zoobotânico de Teresina. Os animais eram alimentados com uma dieta composta por frutas, acelga, ração hiperproteica e água oferecida $a d$ libitum, além de peixes cultivados em lago artificial ao redor do habitat de cativeiro, que eventualmente eram capturados pelos macacos.

Amostras sanguíneas $(5,0 \mathrm{ml}$ de sangue da veia safena lateral) foram coletadas de 15 macacos prego (Cebus sp.) por meio de venopunção. Para tanto, os animais foram contidos física e quimicamente, procurando-se minimizar o estresse no momento da contenção; realizou-se a contenção física através do uso de luvas de couro e puçás. Considerando a idade e o tamanho do animal, a contenção farmacológica instituída foi cloridrato de tiletamina/zolazepam $(7 \mathrm{mg} / \mathrm{kg}$, via IM, em uma única injeção).

A colheita sanguínea foi realizada por meio de seringa e agulha descartáveis, sendo que gotas de sangue foram depositadas em lâminas de vidro para a realização de esfregaço sanguíneo. O restante da amostra sanguínea de cada animal foi distribuído em duas partes, sendo a primeira utilizada para análise bioquímica e a segunda acondicionada em tubos contendo EDTA para análise hematológica. Todos os tubos de coleta foram identificados. Em seguida, lâminas de esfregaço sanguíneo foram coradas por May-Grunwald-Giemsa e analisadas por microscopia de luz.

O restante da amostra foi utilizado para obtenção de perfil hematológico e bioquímico de cada animal. $\mathrm{O}$ exame hematológico foi realizado em analisador hematológico automático Bio 1800 Vet (Bioeasy $\left.{ }^{\circledR}\right)$, com contagem diferencial pelo método manual. O exame bioquímico foi processado em analisador bioquímico semiautomático (Doles D250®). A análise qualitativa dos dados baseou-se em estatística básica (média e desvio padrão).

\section{Resultados e Discussão}

Os animais utilizados neste estudo apresentavamse clinicamente em bom estado de higidez, ativos e alimentando-se adequadamente. A captura e a contenção química dos exemplares foram realizadas conforme as orientações do Manual de vigilância de epizootias em primatas não humanos do Ministério da Saúde (BRASIL, 2005).

$\mathrm{Na}$ análise do material biológico coletado para a realização de esfregaços sanguíneos de cada animal do estudo não foi observado hemoparasitas em sangue periférico. Este resultado difere dos encontrados por Ferreira, Freitas e Molfi (1973) em um estudo realizado no Parque Zoológico da prefeitura de Curitiba, Paraná, Brasil, e Castro et al. (2003) em uma pesquisa realizada com macacosprego de vida livre provenientes de uma reserva semiurbana localizada próximo a cidade de Ribeirão Preto, São Paulo, Brasil, onde foi visualizada a presença de microfilárias em esfregaços sanguíneos de primatas do gênero Cebus.

Com os dados obtidos no exame hematológico, foi possível identificar que todos os animais encontravam-se anêmicos e 46,7\% trombopênicos (SZIRMAI, 1999). Apesar de diversos centros de 
pesquisas e criadouros possuírem primatas do gênero Cebus, ainda há uma grande variação nos valores hematológicos fisiológicos existentes na literatura. As taxas referentes a hemácias, hemoglobina e hematócrito obtidas neste estudo (Tabela 1) estão de acordo com Naves et al. (2006) e com Wirz,
Truppa e Riviello (2008). Entretanto, nos valores de VGM e CHCM existem divergências com aqueles obtidos por Brito Junior et al. (1997), podendo ser explicado pelo estresse agudo, em razão da captura dos animais, e crônico, devido à mudança de recinto vivenciada pelos primatas anteriormente, que influenciam significativamente tais mensurações.

Tabela 1. Média e desvio padrão dos eritrogramas de machos e fêmeas de primatas do gênero Cebus mantidos no Parque Zoobotânico de Teresina (PI) - Teresina - 2012.

\begin{tabular}{lcccccc}
\hline Parâmetros hematológicos & \multicolumn{3}{c}{ Macho } & \multicolumn{3}{c}{ Fêmea } \\
\hline & $\mathrm{N}$ & Média & Desvio padrão & $\mathrm{N}$ & Média & Desvio padrão \\
\cline { 2 - 7 } Hemácias & 06 & 5,35 & 0,33 & 09 & 4,82 & 0,48 \\
Hemoglobina & 06 & 13,63 & 1,08 & 09 & 12,35 & 1,26 \\
Hematócrito & 06 & 37,35 & 2,01 & 09 & 33,25 & 3,01 \\
VGM & 06 & 69,91 & 3,93 & 09 & 69,05 & 1,50 \\
HCM & 06 & 25,40 & 1,05 & 09 & 25,55 & 0,59 \\
CHCM & 06 & 36,43 & 2,33 & 09 & 37,08 & 1,10 \\
Plaquetas & 06 & 349,66 & 98,10 & 09 & 385,00 & 192,08 \\
\hline
\end{tabular}

Fonte: Elaboração dos autores.

Em relação ao sexo dos animais, os resultados encontrados correspondem aos mesmos descritos por Riviello, Wirz (2001) e Costa (2010) onde os machos sobrepujam as fêmeas em relação às hemácias, hemoglobina e hematócrito. Estes parâmetros podem estar associados ao ciclo menstrual das fêmeas, que assim como nos humanos, tem uma diminuição nos dados hematológicos durante a menstruação. Em relação ao VGM, os achados são semelhantes aos encontrados por Costa (2010), pois em ambos, os machos apresentaram um maior valor em relação às fêmeas. Os resultados obtidos para CHGM, também são semelhantes em comparação ao mesmo estudo, onde as fềmeas apresentaram um maior valor. A quantidade de plaquetas foi menor nos machos, entretanto, em ambos os sexos os valores encontrados foram menores que os descritos por Castro et al. (2003).
Quanto ao leucograma, os valores encontrados paraleucócitos, bastonetes, segmentados emonócitos foram maiores que os descritos por Costa (2010), enquanto que a quantidade de eosinófilos, linfócitos e basófilos foram menores. Em um estudo realizado por Riviello e Wirz (2001) com primatas do gênero Cebus clinicamente saudáveis, os resultados foram menores para leucócitos, segmentados, linfócitos e monócitos, entretanto, a quantidade de eosinófilos e basófilos foi maior que as encontradas no presente estudo. A média dos dados relativos à contagem total de leucócitos encontrou-se dentro dos padrões de normalidade, porém comparando-se aos dados existentes na literatura, verificou-se que os valores médios relativos aos monócitos encontravam-se elevados (Tabela 2). Tais valores foram indicativos de que $87 \%$ dos animais apresentavam algum tipo de processo patológico de evolução crônica (SZIRMAI, 1999). 
Tabela 2. Média e desvio padrão do leucograma de machos e fêmeas de primatas do gênero Cebus mantidos no Parque Zoobotânico de Teresina (PI) - Teresina - 2012.

\begin{tabular}{lrrrrrr}
\hline \multicolumn{1}{c}{ Parâmetros hematológicos } & \multicolumn{3}{c}{ Macho } & \multicolumn{3}{c}{ Fêmea } \\
\hline & $\mathrm{N}$ & Média & Desvio padrão & $\mathrm{N}$ & Média & Desvio padrão \\
\cline { 2 - 6 } Leucócitos Totais & 06 & 12,18 & 5,20 & 09 & 10,87 & 2,70 \\
Bastonetes & 06 & 0,00 & 0,00 & 09 & 0,11 & 0,33 \\
Segmentados & 06 & 46,70 & 17,40 & 09 & 51,00 & 9,43 \\
Eosinófilos & 06 & 0,30 & 0,50 & 09 & 1,33 & 1,11 \\
Basófilos & 06 & 0,30 & 0,50 & 09 & 0,00 & 0,00 \\
Linfócitos & 06 & 40,00 & 16,40 & 09 & 33,22 & 11,00 \\
Monócitos & 06 & 12,70 & 4,70 & 09 & 14,33 & 5,43 \\
\hline
\end{tabular}

Fonte: Elaboração dos autores.

Houve diferenças nos resultados encontrados no presente estudo em comparação aos obtidos por Riviello, Wirz(2001) em relação ao sexo dos animais. Os machos apresentaram uma maior quantidade de leucócitos totais, linfócitos e basófilos, enquanto que as fêmeas obtiveram uma maior quantidade de bastonetes, segmentados, eosinófilos e monócitos.

Realizou-se a mensuração dos índices bioquímicos (Tabela 3), tais como ureia, creatinina, fosfatase alcalina e transaminases (ALT e AST). Os valores de ureia foram superiores nas fêmeas, diferindo dos resultados apresentados por Larsson et al. (1997) e creatinina em ambos os estudos, os machos obtiveram valores maiores. A fosfatase alcalina foi mais elevada nos machos, porém, no presente estudo este parâmetro estava mais de $100 \%$ acima dos valores relatados na literatura como normais para ambos os sexos. AALT e AST estavam maiores nas fêmeas, mas também apresentaram valores acima dos considerados normais para o gênero Cebus (SZIRMAI, 1999). Tais parâmetros bioquímicos alterados da função hepática, tanto pela fosfatase alcalina quanto ALT e AST são indicadores de injúria do parênquima hepático.

Tabela 3. Média e desvio padrão dos valores bioquímicos de machos e fêmeas de primatas do gênero Cebus mantidos no Parque Zoobotânico de Teresina (PI) - Teresina - 2012.

\begin{tabular}{lrrrrrr}
\hline \multicolumn{1}{c}{ Parâmetros Bioquímicos } & \multicolumn{3}{c}{ Macho } & \multicolumn{2}{c}{ Fêmea } \\
\hline & $\mathrm{N}$ & Média & Desvio padrão & $\mathrm{N}$ & \multicolumn{1}{c}{ Média } & Desvio padrão \\
\cline { 2 - 7 } Ureia & 06 & 38,85 & 8,41 & 08 & 43,64 & 21,31 \\
Creatinina & 06 & 0,95 & 0,21 & 08 & 0,93 & 0,16 \\
ALP & 05 & 14,34 & 7,84 & 08 & 29,65 & 48,76 \\
ALT & 06 & 39,07 & 48,18 & 08 & 60,43 & 74,77 \\
Fosfatase Alcalina & 05 & 424,74 & 200,62 & 05 & 302,92 & 202,55 \\
\hline
\end{tabular}

Fonte: Elaboração dos autores.

Em adição, ao analisarmos em conjunto os valores eritrocitários e o número de monócitos em associação com as mensurações elevadas das enzimas hepáticas, seria lícito sugerir que a sanidade dos macacos-prego pertencentes ao Parque Zoobotânico esteja comprometida. Sabe-se que doenças degenerativas crônicas como Hepatite A raramente mostram sinais clínicos evidentes de doença em primatas não humanos, mas apresentam níveis elevados de enzimas hepáticas (Fosfatase Alcalina e ALT) associadas à anemia e tempo de coagulação aumentado (SZIRMAI, 1999). 
Dados do Ministério da Saúde revelam que primatas não humanos neotropicais do gênero Cebus, em condições de infecção natural, são responsáveis pela ocorrência de epizootias, o que inclui a hepatite A em várias regiões do Brasil após contaminação com o vírus (BRASIL, 2005). Nas doenças zoonóticas é possível à transmissão de patógenos como o vírus da hepatite $\mathrm{A}$ dos homens aos animais, podendo ocorrer também o inverso, o que se caracteriza como fator de perpetuação de doenças na criação de primatas em cativeiro, com implicações para a saúde pública (PEREIRA et al., 2010).

A hepatite A apresenta-se como uma zoonose ocasionada por um picornavirus, que possui como hospedeiros naturais o homem e primatas não humanos. A infecção ocorre pela via oro-fecal e o vírus é eliminado nas fezes após replicação no fígado. Ainda, dos primatas não humanos mantidos em cativeiro, estudos estimam que entre $4,0 \%$ e $7,6 \%$ dos animais estiveram em contato com o vírus da hepatite $\mathrm{A} e$, quanto maior o contato entre humanos e esses animais, maior a prevalência de hepatite A em primatas não humanos (SETZER et al., 2002). Como os sinais clínicos em primatas são geralmente assintomáticos e quando presentes são inespecíficos, a possibilidade de no Parque Zoobotânico haverem animais infectados e não diagnosticados é bastante elevada.

Doenças zoonóticas podem estar sendo propagadas por primatas não humanos mantidos em cativeiro e, portanto, estudos sobre a sanidade desses animais são de fundamental importância para a Saúde Pública, além de permitir uma melhor compreensão da linha tênue que separa o estado de Saúde e de Doença.

Desta feita, tornam-se necessários estudos futuros, como ensaios sorológicos e de reação em cadeia de polimerase (PCR) para melhor elucidar o verdadeiro estado de sanidade dos primatas não humanos pertencentes ao Parque Zoobotânico de Teresina para, assim, propor ações preventivas que reflitam na Saúde Pública.

\section{Observação}

Este estudo obteve autorização para atividade com finalidade científica junto ao Sistema de Autorização e Informação em Biodiversidade (SISBIO No 26302-1), e aprovado pelo Comitê de Ética em Experimentação Animal ( ${ }^{\circ}$ 011/11).

\section{Referências}

ACHA, P. N.; SZYFRES, B. Zoonosis y enfermidades transmisibles comunes al hombre y animales. 3. ed. Washington: Organization Panamericana de la Salud, 2003.

AMÂNCIO, F. H.; SANTOS, A. C. B.; PRINCE, K. A.; CRUVINEL, T. M. A.; CRUVINEL, C. A. T.; GUERRA NETO, G.; SILVA, C. S.; PACHALY, J. R.; LEITE, C. Q. F.; PANDOLFI, J. R. Isolamento de mycobacterium lentiflavum em primatas não humanos mantidos em cativeiro. In: CONBRAVET, 35., 2008, Gramado. Anais eletrônicos... Gramado: SBMV, 2008. Disponível em: $<$ http://www.sovergs.com.br/conbravet2008/anais/cd/ resumos/R1298-2.pdf>. Acesso em: 02 fev. 2013.

BRASIL. Ministério da Saúde. Secretaria de Vigilância em Saúde. Manual de vigilância de epizootias em primatas não-humanos. Brasília: Ministério da Saúde, $2005.56 \mathrm{p}$.

BRITO JÚNIOR, L. C.; MUNIZ, J. C. P. C.; MARTINS, N. Y.; AZEVEDO, P. S. R. Valores hematológicos do macaco prego, Cebus apella (L., 1758), reproduzido e mantido em cativeiro. Revista Brasileira de Medicina Veterinária, v. 19, n. 5, p. 188-192, 1997.

BURGOS-RODRIGUES, A. G. Zoonotic diseases of primates. Veterinary Clinics of North America: Exotic Animal Pratice, Philadelphia, v. 14, n. 3, p. 557-575, 2011.

CARMO, A. M.; SALGADO, C. A. Ocorrência de parasitos intestinais em Callithrix sp.(Mammalia, Primates, Callithrichidae). Revista Brasileira de Zoociência, Juiz de Fora, v. 5, n. 2, p. 267-272, 2003.

CASTRO, M. P.; NAKAGE, A. P. M.; BARBANTE, P.; AMARAL, J. M. G.; DE JONG, D.; MARINHEIRO, M. T.; HOPPE, E. G. L.; GUERRA NETO, G.; ANDRADE, T. M.; RODRIGUES, R. Avaliação hematológica de macacos-prego (Cebus apella, Linnaeus, 1758) de vida livre. In: CONGRESSO, 7.; ENCONTRO DA ABRAVAS, 12., 2003, São Pedro. Anais... São Pedro: ABRAVAS, 2003. p. 50-51. 
CENTERS FOR DISEASE CONTROL AND PREVENTION - CDC. Compendium of measures to prevent disease associated with animals in public settings. Atlanta: MMWR, 2009. 21 p.

CORRÊA, S. H. R.; PASSOS, E. C. Wild animals and public health. In: FOWLER, M. E.; CUBAS, Z. S. Biology, medicine, and surgery of South American wild animals. Ames: Iowa University Press, 2001. p. 493-499.

COSTA, S. M. Frequência de anticorpos contra leptospira spp. e trypanosoma cruzi em primatas neotropicais mantidos em cativeiro. 2010. Dissertação (Mestrado em Ciência Animal) - Núcleo de Ciências Agrárias e Desenvolvimento Rural. Universidade Federal do Pará, Belém.

FERREIRA, D. S.; FREITAS, O. T.; MOLFI, A. Hemocitologia de cebus cerrifis. Acta Biológica Paranaense, Curitiba, v. 2, n. 1-4, p. 9-15, 1973.

LARSSON, M. H. M. A.; LUCAS, S. R. R.; MIRANDOLA, R. M. S.; LAZARETTI, P.; FEDULHO, J. D. L.; GUIMARÃES, M. A. B. V. Valores de referência das provas de funções hepática, renal e de alguns eletrólitos em Cebus apella, anestesiados com cetamina. Ciência Rural, Santa Maria, v. 27, n. 2, p. 257-262, 1997.

LEITE, T. N. B.; MAJA, T. A.; OVANDO, T. M.; CANTADORI, D. T.; SCHIMIDT, L. R.; GUÉRCIO, A. C.; CAVALCANTI, A.; LOPES, F. M. R.; CUNHA, I. A.L.; NAVARRO, I. T. Ocorrência de infecção por Leishmania spp. e Toxoplasma gondii em macacos-prego (Cebus apella) de Campo Grande, MS. Revista Brasileira de Parasitologia Veterinária, Jaboticabal, v. 17, n. 1, p. 307-310, 2008.

MONTEIRO, R. V. Zoonoses de animais silvestres. Ciência Veterinária nos Trópicos, Recife, v. 11, p. 170173, abr. 2008. Suplemento 1.
NATIONAL ASSOCIATION OF STATE PUBLIC HEALTH VETERINARIANS - NASPHV. Compendium of veterinary standard precautions for zoonotic disease prevention in veterinary personnel. Saint Paul: JAVMA, 2008. $15 \mathrm{p}$.

NAVES, E. A.; FERREIRA, F. A.; MUNDIM, A. V.; GUIMARÃES, E. C. Valores hematologicos de macaco prego (Cebus apella - Linnaeus, 1758) em cativeiro. Bioscience Journal, Uberlândia, v. 22, n. 2, p. 125-131, 2006.

PEREIRA, W. L. A.; GALO, K. R.; SILVA, K. S. M.; SOARES, M. C. P.; ALVES, M. M. Ocurrencia de hepatitis virales, helmintiasis y protozoosis em primates neotropicales procedentes de cria domiciliaria: afecciones de transmisión fecal-oral com potencial zoonótico. Revista Pan-Amazônica de Saúde, Ananindeua, v. 1, n. 3, p. 57-60, 2010.

RIVIELLO, M. C.; WIRZ, A. Haematology and blood chemistry of cebus apella in relation to sex and age. Journal of Medical Primatology, Malden, v. 30, n. 6, p. 308-312, 2001.

SETZER, A. P.; PISSINATYI, A.; GASPAR, A. M. C.; SIDONI, M.; DIAS, J. L. C. Soroprevalência de hepatite A em primatas neotropicais. In: CONGRESSO E XI ENCONTRO DA ABRAVAS, 6., 2002, Guarapari. Anais... Gurapari: ABRAVAS, 2002. p. 46.

SZIRMAI, A. G. K. Clínica e terapêutica em primatas neotropicais. Juíz de Fora: UFJF, 1999. 264 p.

WIRZ, A.; TRUPPA, V.; RIVIELLO, C. Hematological and plasma biochemical values for captive tufted capuchin monkeys (Cebus apella). American Journal of Primatology, v. 70, n. 5, p. 463-472, 2008.

WOLFE, N. D.; ESCALANTE, A. A.; KARESH, W. B.; KILBOURN, A.; SPIELMAN, A.; LAL, A. A. Wild primate population in emerging infectious disease research: the missing link? Emerging Infectious Diseases, Atlanta, v. 4, n. 2, p. 149-158, 1998. 
